\title{
Editorial: Image-Guided Radiotherapy for Effective Radiotherapy Delivery
}

\author{
Nam P. Nguyen ${ }^{1 *}$ and Ulf Lennart Karlsson ${ }^{2}$ \\ ${ }^{1}$ Department of Radiation Oncology, Howard University, Washington, DC, USA, ${ }^{2}$ Department of Radiation Oncology, \\ Marshfield Clinic, Marshfield, WI, USA
}

Keywords: cancer, computerized axial tomography, image-guided radiotherapy, comorbidity, disease-specific survival

During most of the last century, verification of patient position on the radiotherapy treatment table was considered adequate if exposed on a photographic film by a megavoltage beam. It was a general standard to expose such a film once a week, to be approved by a radiation oncologist. The latter approved it after comparison to a kilovoltage simulation film exposed at the time of initial setup of the patient before the treatment regimen started.

A common rule was to allow a $\pm<5 \mathrm{~mm}$ variation from the simulation to the treatment portal film. This often resulted in either an approval for the next week's treatment fractions or a rejection and retake of that or the next day's portal film. There was no film record of the next four fractions. The problems included megavoltage film resolution judged from kilovoltage simulation films as well as unrecorded possible errors for the next four fractions. Another error source was soft tissue contrast in both of these films.

The evolution of computerized axial tomography (CAT) scan from the mid-twentieth century has allowed for $3 \mathrm{D}$ reconstruction of the patient's soft tissue structures by improved resolution in millimeter scan slices.

Development of the digital image visualization on computer screens now allows for fusing the reconstructed simulation image (DRR) from the CAT scanner with the mega- or kilovoltage rendering of the patient's treatment beams. This has allowed the skilled radiotherapist to adjust the beam within a preset millimeter 3D frame to the patient's anatomy. With this precision, a daily treatment fraction is given. The radiation oncologist can then check that body position errors have been corrected before each treatment.

Further improvement include the cone beam image obtained from the treatment accelerator and fused over the DRR, introduction of gold markers in the target volume and triangulating their positions into the simulation scan, as well as utilizing kilovoltage and or megavoltage images to attain precise beam geometry for each daily radiotherapy fraction. Another method is to use a diagnostic CAT scanner that is mechanically attached to the accelerator.

These imaging techniques are used to assure that the planned dose only covers the intended target and encompasses the IGRT concept in radiotherapy. If used properly, the precision of treatment is improved from centimeter to millimeter realms (1) and is expected to be used globally in cancer radiotherapy. Our experience is that few treatment portals need to be rejected as long as there is a requirement of immediate report to the oncologist that a specified position error has been discovered and corrected.

We consider it a necessary ingredient for clinical studies in order to measure and compare IGRT outcome data. It has the potential of not only providing better toxicity results but also to give better outcome data for patient groups who are thought to be at higher risk for toxicity, e.g., frail elderly and patients with abnormal radiosensitivity. It may also offer an avenue for dose escalation because of better organ sparing. 
Our preliminary evidence is encouraging for the use of IGRT.

Elderly ( $>70$ years of age) and younger head and neck cancer groups both tolerated definitive chemo-IGRT, without difference in grade 3-4 toxicity, treatment breaks, and with less weight loss in the elderly group (2). Another study resulted in disease-specific survival of $75 \%$ at 4 years and acceptable toxicity (3).

Elderly patients with multiple comorbidities and locally advanced rectal cancer tolerated preoperative chemo-IGRT when compared to younger patients (4). These preliminary studies suggest that IGRT may become the treatment of choice for elderly cancer patients.

Another subset of patients who may benefit from IGRT is patients with human immunodeficiency virus (HIV) infection and anal cancer. They may have an increased sensitivity to radiation because of thiol deficiency (5). Grade 3-4 skin, hematologic and gastrointestinal toxicity were frequent among HIV positive patients undergoing standard chemoradiotherapy and may result in death $(6,7)$. Chemo-IGRT may therefore

\section{REFERENCES}

1. Oehler C, Lang S, Dimmerling P, Bolesch C, Kloeck S, Tini A, et al. PTV margin definition in hypofractionated IGRT of localized prostate cancer using cone beam CT and orthogonal image pairs with fiducial markers. Radiat Oncol (2014) 9:229. doi:10.1186/s13014-014-0229-z

2. Nguyen NP, Vock J, Chi A, Vinh-Hung V, Dutta S, Ewell L, et al. Impact of intensity-modulated and image-guided radiotherapy on elderly patients undergoing chemoradiation for locally advanced head and neck cancer. Strahlenther Onkol (2012) 188:677-83. doi:10.1007/s00066-012-0125-0

3. Bahig H, Fortin B, Alizadeth M, Lambert L, Filion E, Guertin L, et al. Predictive factors of survival and treatment tolerance in older patients treated with chemotherapy and radiotherapy for locally advanced head and neck cancer. Oral Oncol (2015) 51(5):521-8. doi:10.1016/j.oraloncology.2015.02.097

4. Nguyen NP, Ceizyk M, Vock J, Vos P, Chi A, Vinh-Hung V, et al. Feasibility of image-guided radiotherapy for elderly patients with locally advanced rectal cancer. PLoS One (2013) 8:e71250. doi:10.1371/journal.pone.0071250

5. Vallis KA. Glutathione deficiency and radiosensitivity in AIDS patients. Lancet (1991) 337:918-9. doi:10.1016/0140-6736(91)90250-S

6. Alfa-Wali M, Allen-Mersh T, Antoniou A, Tait D, Newsom-Davis T, Gazzard $\mathrm{B}$, et al. Chemoradiotherapy for anal cancer in HIV patients causes prolonged CD4 cell count suppression. Ann Oncol (2012) 23:141-7. doi:10.1093/annonc/ mdr050

7. Cleator S, Fife K, Nelson M, Gazzard B, Phillips R, Bower M. Treatment of HIVassociated invasive anal cancer with combined chemoradiation. Eur J Cancer (2000) 36:754-8. doi:10.1016/S0959-8049(00)00009-5

8. Nguyen NP, Vock J, Sroka T, Khan R, Jang S, Chi A, et al. Feasibility of image-guided radiotherapy based on tomotherapy for the treatment of locally advanced anal cancer. Anticancer Res (2011) 31:4393-6. provide HIV patients the opportunity to be treated with less toxicity $(8,9)$.

Finally, IGRT may allow for radiation dose escalation in cancers with high-risk for loco-regional recurrences. A recent randomized study reported a 2-year survival of 57 and $44 \%$ and local failure of 30 and $38 \%$ for locally advanced NSCLC treated to 60 and $74 \mathrm{~Gy}$, respectively. The poor survival in the 74 Gy group may be associated with cardiac toxicity (10).

A 3-year survival of $45 \%$ and local failure of $15 \%$ was reported for patients with locally advanced NSCLC treated to 70-75 Gy with chemo-IGRT, with minimal toxicity (11). Dose escalation was also feasible in patients with locally advanced esophageal cancer because of lung and cardiac sparing (12).

These preliminary results are intriguing but need to be corroborated in future prospective studies.

\section{AUTHOR CONTRIBUTIONS}

UK and NN wrote and approved the manuscript.

9. Nguyen NP, Ceizyk M, Almeida F, Chi A, Betz M, Moderrasifar H, et al. Effectiveness of image-guided radiotherapy for locally advanced rectal cancer. Ann Surg Oncol (2011) 18:380-5. doi:10.1245/s10434-010-1329-0

10. Bradley J, Paulus R, Komaki R, Masters G, Blumenschen G, Schild S, et al. Standard dose versus high dose conformal radiotherapy with concurrent and consolidation carboplatin plus paclitaxel with and without cetuximab for patients with stage IIIA and IIIB non-small cell lung cancer (RTOG 0617): a randomized, two-by-two factorial phase II study. Lancet Oncol (2015) 16: 187-99. doi:10.1016/S1470-2045(14)71207-0

11. Nguyen NP, Kratz S, Chi A, Vock J, Vos P, Shen W, et al. Feasibility of imageguided radiotherapy and concurrent chemotherapy for locally advanced nonsmall cell lung cancer. Cancer Invest (2015) 33:53-60. doi:10.3109/07357907. 2014.1001896

12. Nguyen NP, Jang S, Vock J, Vinh-Hung V, Chi A, Vos P, et al. Feasibility of intensity-modulated and image-guided radiotherapy for locally advanced esophageal cancer. BMC Cancer (2014) 14:265. doi:10.1186/14712407-14-265

Conflict of Interest Statement: The authors declare that the research was conducted in the absence of any commercial or financial relationships that could be construed as a potential conflict of interest.

Copyright (C) 2015 Nguyen and Karlsson. This is an open-access article distributed under the terms of the Creative Commons Attribution License (CC BY). The use, distribution or reproduction in other forums is permitted, provided the original author(s) or licensor are credited and that the original publication in this journal is cited, in accordance with accepted academic practice. No use, distribution or reproduction is permitted which does not comply with these terms. 